\title{
A Research on Application of Human-Robot Interaction using Artifical Intelligence
}

\author{
L.Mary Gladence, Vakula C.K, Mercy Paul Selvan, T.Y.S.Samhita
}

\begin{abstract}
This work presents an online robot instructing with common human - robot connection. Normal human-PC association is a critical interface to acknowledge benevolent joint effort of canny robot and human. The greater part of the correspondence between people is done through discourse and signal, and the connection among discourse and motion is regular and natural. Robot educating by methods for discourse acknowledgment [12] is another route for instructing and playback, which utilizes the common discernment channels of individuals. This paper centers around a training technique dependent on the common human-PC communication. The task is to teach the Robot to compose by giving three unique information sources like Voice order, Camera based Video info or utilizing MEMS equipment interface utilizing Zigbee. Voice direction can be perceived utilizing Android application. Motion will perceived utilizing framework camera, utilizing PCA calculation framework will order the pictures. MEMS sensor is wired equipment hardware which will contain the quantity of likelihood blend to order the robot. Motions are segregated by applying a most extreme data model, with highlights separated utilizing principal component analysis (PCA). The proposed interface could be stretched out to the genuine modern scene. By utilizing signal and discourse, administrators can control the robot without complex tasks. The outcomes show that the online robot instructing framework can effectively show robot controllers.
\end{abstract}

Keywords: Gesture, human-robot interaction, PCA, natural speech understanding, online robot teaching.

\section{INTRODUCTION}

Powerful collaboration among people and robots in any condition, and in any methods for correspondence, depends vigorously on the unambiguous trade of data. On account of human-to-human correspondence, verbally expressed discourse is the overwhelming strategy for collaboration, however motion based correspondence is genuinely normal, especially in situations where discourse based correspondence is beyond the realm of imagination (e.g., being submerged, or having some type of correspondence incapacitate). For across the board arrangement of robots in the general public, an errorfree, strong and common strategy for human-robot collaboration is essential [21,22].

An elective methodology is outline directions from a human client to an expectation in a given errand setting. With regards to this paper, expectation is the target the client needs the robot to accomplish. Applications in discourse

Revised Manuscript Received on July 18, 2019.

L.Mary Gladence, Assistant professor, Dept. of IT, Sathyabama Institute of science and Technology, Chennai,Tamilnadu, India.

Vakula C.K, UG Student, Dept. of IT, Sathyabama Institute of Science and Technology, Chennai, Tamilnadu, India.

Mercy Paul Selvan, Assistant professor, Dept. of IT, Sathyabama Institute of science and Technology, Chennai,Tamilnadu, India.

T.Y.S.Samhita, UG Student, Dept. of IT, Sathyabama Institute of Science and Technology, Chennai, Tamilnadu, India. acknowledgment, normal dialect handling, and machine learning have made it feasible for a PC to decide the purpose of an order issued by a human, while techniques from human-robot cooperation (HRI) have made it workable for robots to give helpful criticism to these directions given their condition. Our objective in this paper is to exhibit a system for characteristic dialect. With the advancement of industry 4.0, robots later on will in general be canny, multifunctionalized and community oriented. In this manner, it is essential to understand the correspondence among people and robots. With the entry of the period of knowledge, individuals started to anticipate the more elevated amount of interest for wise innovation [1], which drives robots to create towards the heading of insight and enhancement. Human robot collaboration [2] is a developing field in the examination of robot innovation. The start and establishment of the human PC collaboration innovation are the robot educating [3] and playback innovation [4], which implies that human show robots information somehow or another. The robot has the capacities of getting the hang of, recalling, and seeing, which incorporated the advancement aftereffects of numerous controls.

All through the improvement of robot educating playback innovation, there are three primary bearings: utilizing a joystick [5] [6], showing dependent on discourse acknowledgment [7] [8] and utilizing an instruct pendant. The standards, focal points and drawbacks of the three bearings are unique. The standard of joystick educating playback strategies is to see the spatial area data of the joystick, at that point imitate the changing procedure of these positions. In past examinations, various specialists have received joysticks and haptic gadgets to educate and control the robots.

In [9], a strategy for robot educating and rendering by wearing a metal skeleton is presented. This playback mode is especially appropriate for humanoid robot, and the utility model has the benefits of long separation control and no prerequisite of workplace. Furthermore, due to the adaptability of humanoid structure, this mode can total some increasingly mind boggling work. In [10] [11], the omega haptic gadgets are utilized to control the robot arm..In this framework, in any case, the instruments (i.e., the joystick and the haptic gadget) limit the workspace.

Regular human-PC connection is a critical interface to acknowledge inviting coordinated effort of wise robot and human. A large portion of the correspondence between people is done through discourse and motion, and the collaboration among discourse and motion is characteristic

Published By:

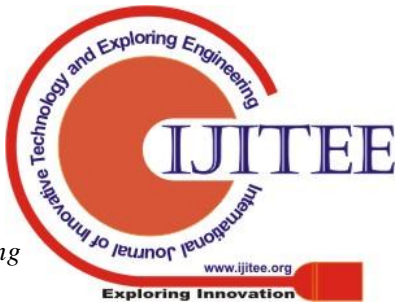


and natural. Robot educating by methods for discourse acknowledgment [12] is another route for instructing and playback, which utilizes the common recognition channels of individuals. This paper centers around an instructing technique dependent on the common human-PC communication

Contrasted and recently created show strategies, movement sensor innovation can be utilized in instructing playback robot controller framework. In [13], the Wii remote controller perceives human movement and controls the 3-D development and revolution of the robot arm. In [14], another movement way instructing framework is proposed.

\section{RELATED WORK}

The grouping and acknowledgment of new visual items have been concentrated seriously from visual discernment and machine learning points of view have been referred from [1]-[20]. Numerous methodologies, for example, the sacks of visual words we are utilizing in this paper, have been as of late created [15]. Those learning frameworks are exceptionally proficient when prepared with a huge database of good marked models (see, for example, PASCAL VOC). However, to take care of this issue in a genuine HRI situation, i.e., outside of the lab, with nonexpert clients in a sensible use situation, one needs to handle a pivotal issue not tended to in the machine learning and PC vision writing: For example, they are firmly identified with research done in robot dialect obtaining and specifically the development of outwardly grounded vocabularies

For example, pointing motions and discourse acknowledgment, to allowusers to teachwords related with articles to anAIBO robot [17]. Scasselati utilized guiding signals and look following toward draw a robot's consideration [18].

In this investigation, he utilized a settled upper middle and, along these lines, obliged the communication. Pointing motions have likewise been utilized to manage a robot buddy.Sadly, existing related systems for motion, look and discourse acknowledgment, and elucidation are not sufficiently strong in uncontrolled conditions (because of commotion, lighting, or impediment).Roy displayed a structure that enables an automated framework to secure outwardly grounded words. In their work, Kaplan and Steels recognized the absence of power in the interface as a noteworthy impediment of their framework and demonstrated that the absence of vigor of the interface regularly prompts various terrible learning models.

Another broadly utilized approach to handle this pointing and joint consideration issue is to enable clients to straightforwardly wave protests before the camera of the robot .Therefore, we can ask the robot to dependably concentrate on the moving items. For example, Kemp et al. utilized a laser pointer to effectively and vigorously assign items to a robot so as to request that it get those. Here, they utilized the laser pointer as a point-and-snap interface. They demonstrated that unpracticed members figured out how to accurately assign items to a robot.

\section{ONLINE ROBOT TEACHING SYSTEM}

As indicated by the qualities of intelligent mode, the two methods of unpleasant educating and fine instructing are characterized (Fig. 1), in order to enhance the effectiveness while guaranteeing the prerequisite of exactness. This sort of undertaking requires less exactness, for example, moving toward targets and evasion programs on the other hand, the undertakings with a high accuracy require a tweaking of the position or introduction close to the objective position. The blend of discourse and static signals is expected to control. with versatile subspace, which considers total steady learning.

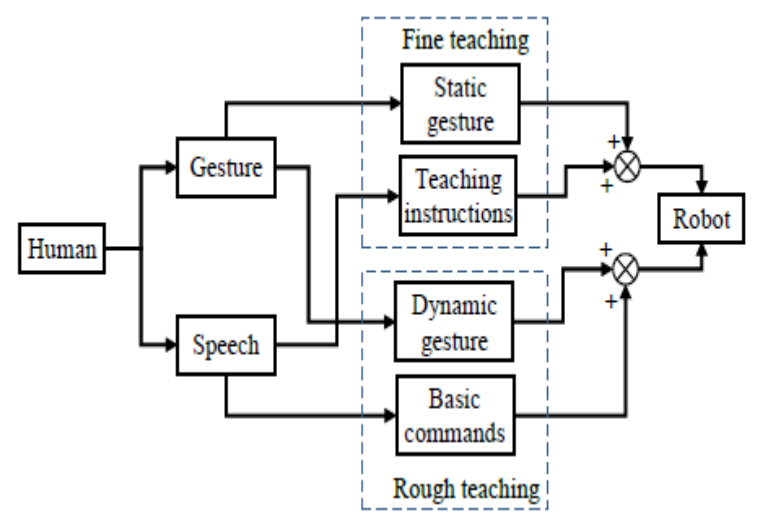

Fig1Online robot teaching system process.

\section{Existing System}

The instructing strategy is never again appropriate for the creation mode with human - robot joint effort. A profundity camera (Kinect) and an inertial estimation unit (IMU) are utilized to catch the discourse and signal of the human Maximum entropy calculation is utilized to manage the combination content into the comparing robot guidelines. Interim Kalman channel and enhanced molecule channel are utilized to gauge the signal. To incorporate discourse and motion data all the more profoundly, a novel strategy for broad media combination dependent on content (AVFT) is proposed, which can remove the most helpful data from the discourse and signals by changing them into content.

\subsection{Disadvanatges Of Existing System}

The conventional training technique has the weaknesses of moderate speed, high time cost and the program is excessively confused. These detriments make it hard to be equipped later on generation mode. Other than the time cost, sensor cost is additionally a factor confining the improvement of robots.

\section{Proposed system}

The proposed work empowers individuals and robots to convey through common dialect. A web based showing strategy with the combination of discourse and motion this application is characterized by the teacher to control the robot. To train the Robot to compose by giving three distinct information sources like Voice order; Camera based video 
info or utilizing MEMS equipment interface utilizing Zigbee. Voice order can be perceived utilizing Android application. This new framework is made out of a training pen and a movement catch framework.

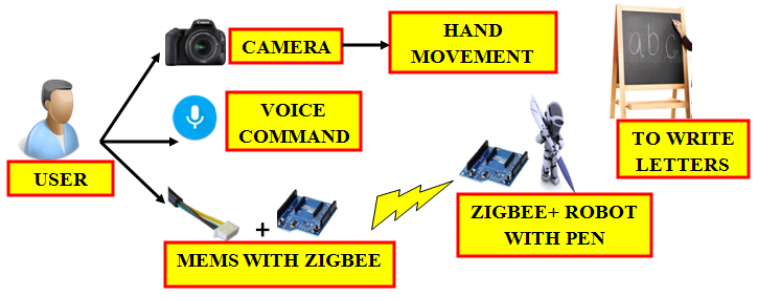

Fig 2 Overview ofProposed System

In this venture the PCA calculation is utilized to motion acknowledgment of discourse and motion acknowledgment dependent on online PCA calculation with versatile subspace, which takes into consideration complete steady learning. The upside of this technique is changing over the discourse data and signal data into writings to accomplish integral focal points. The piece of online PCA learning is the key purpose of this framework. The new mean vector, new eigenvalues, new eigenvectors and new subspace are acquired through continuous web based showing technique with the combination of discourse and motion the regular intuitive instructing strategies utilize shabby sensor to acknowledge web based educating for the errands. At last, the assessment of the strategy is made by contrasting and the current techniques.

\section{SYSTEM DESIGN \& RESULTS}

This area for the most part presents how the robots comprehend complex content, which is combination content including discourse content and signal content. In light of the three issues above, we proposed a structure of an online robot educating framework. The system comprises of three sequential layers: input layer, association layer and yield layer.

\subsubsection{Gesture Recognition}

In this module we train the PC to perceive the signal which is given by the client. In light of motion robot will perceive the sign and react dependent on that like forward, in reverse development. For this we use PCA calculation to recognize the sort of signal given by the client. We train the framework by giving arrangement of motion utilizing Matlab. After that PCA calculation will arrange the pictures and send it to the robot by means of equipment.

\subsubsection{Android Voice Command}

In this module client will give order by means of versatile application as a voice. In light of voice direction robot will react. Utilizing android studio we make an android application to perceive the voice. Through Google voice acknowledgment we catch the order and send it to the robot.

\subsubsection{Hardware Interface}

Implanted equipment gadgets is developed utilizing PIC board and MEMS sensor. MEMS sensor is a wired gadget to perceive the development that is tilting of wire by directly to left, left to ideal, forward to in reverse and in reverse to forward. For this additionally we will prepare the robot to react dependent on shaking of mems.

\subsubsection{Wireless Communication}

Zigbee is an IEEE 802.15.4-based determination for a suite of abnormal state correspondence conventions used to make individual territory systems with little, low-control advanced radios, for example, for home computerization, medicinal gadget information gathering, and other lowcontrol low-data transfer capacity needs, intended for little scale ventures which require remote association. Thus, Zigbee is a low-control, low information rate, and nearness (i.e., individual territory) remote specially appointed system.

\subsubsection{Writing Robot}

In light of voice order and signal robot will compose a solitary character and for correspondence we use Zigbee which is remote correspondence to speak with robot.

\section{CONCLUSION}

In this paper showed an effective and dependable framework for web based learning and acknowledgment of signals. Web based learning of signals in an intuitive framework can be utilized to make collaboration among robots and people less demanding in applications. This paper centers around the robot training application based $n$ the characteristic human-robot communication. This paper displays an online robot instructing strategy that wires discourse and motion. This strategy permits the staffs who need instructing aptitudes to play out the assignment of robot educating rapidly and adequately

\section{REFERENCES}

1. B. Gates. (2007, Jan.). "A robot in every home," Sci. Amer. [Online].Available: http://www.sciam.Com/article.cfm? Id=a-robot-in-everyhome

2. T. W. Fong, I. Nourbakhsh, and K. Dautenhahn, "A survey of sociallyinteractive robots," Robot. Auton. Syst., vol. 42, pp. 143-166, 2003.

3. C. L. Nehaniv and K. Dautenhahn, Eds., Imitation and Social Learning inRobots, Humans, and Animals: Behavioral, Social and CommunicativeDimensions. Cambridge, U.K.: Cambridge Univ. Press, 2004.

4. A. Billard, S. Calinon, R. Dillmann, and S. Schaal, "Survey: Robot programmingby demonstration," Handbook of Robotics.NewYork: Springer,2008, ch. 59.

5. A. L. Thomas and C. Breazeal, "Teachable robots: Understanding humanteaching behavior to build more effective robot learners," Artif. Intel. J.,vol. 172, pp. 716-737, 2008.

6. A. Thomas and C. Breazeal, "Robot learning via socially guided exploration,"in Proc. IEEE 6th Int. Conf. Dev. Learn., Jul. 2007, pp. 82-87.

7. S. Calinon, F. Guenter, and A. Billard, "On learning, representing, andgeneralizing a task in a humanoid robot," IEEE Trans. Syst., Man, Cybern.B, Cybern. vol. 37, no. 2, pp. 286-298, Apr. 2007.

8. P. Miller, Theories of Developmental Psychology, 4th ed. New York:Worth, 2001 
9. L.Mary Gladence,Karthi,M.,Ravi, T “A novel technique for Multi-class ordinal regression-APDC" IndianJournal of Science and Technology Vol.9/ No.10/March 2016/1-5

10. M. Tomasello, M. Carpenter, J. Call, T. Behne, and H. Moll, "Understandingand sharing intentions: The origins of cultural cognition," Behav.Brain Sci., vol. 28, no. 5, pp. 675-690, 2005.

11. F. Kaplan and V. Hanker. (2004). the challenges of joint attention. Proc.4th. Int. Workshop Epigenetic Robotics [Online]. pp. 67-74. Available:http://cogprints.org/4067/

12. L. Steels and F. Kaplan, "Aibo's first words: The social learning of languageand meaning," Evol. Commun., vol. 4, no. 1, pp. 3-32, 2000.

13. K. Dautenhahn and J. Saunders. (2011). New Frontiers in Human-Robot Interaction (Advances in Interaction Studies Series). Amsterdam,The Netherlands: John Benjamin's. [Online]. Available: http://books.google.fr/books?id=_FIP3ZBhq6oC

14. P.-Y. Oudeyer. (2011). "Developmental robotics," in Encyclopedia of theSciences of Learning (Springer Reference Series), N. Seel, Ed. New York:SpringerVerlag [Online]. Available: http://hal.inria.fr/hal00652123

15. K. Ishii, S. Zhao, M. Inami, T. Igarashi, and M. Imai, "Designing lasergesture interface for robot control," in Proc. 12th IFIP Conf. Human-Comput. Interact., 2009, pp. 479-492.

16. P. Rouanet, P.-Y. Oudeyer, and D. Filliat, "An integrated system for teachingnew visually grounded words to a robot for non-expert users using amobile device," in Proc. IEEE-RAS Int. Conf. Humanoid Robots, 2009,pp. 391-398.

17. P. Rouanet, P. Oudeyer, and D. Filliat, "Using mediator objects to easilyand robustly teach visual objects to a robot," in Proc. ACM SIGGRAPHPosters, 2010.

18. Gladence, L. Mary, Hari Haran Sivakumar, Gobinath Venkatesan, and S. Shanmuga Priya. "Home and office automation system using human activity recognition." In 2017 International Conference on Communication and Signal Processing (ICCSP), pp. 0758-0762. IEEE, 2017

19. P. Rouanet, F. Danieau, and P.-Y. Oudeyer, "A robotic game to evaluateinterfaces used to show and teach visual objects to a robot in real worldcondition," in Proc. 6th Int. Conf. Human-Robot Interact., 2011, pp. 313-320.

20. Gladence, L. Mary, M. Karthi, and V. Maria Anu. "A statistical comparison of logistic regression and different Bayes classification methods for machine learning." ARPN Journal of Engineering and Applied Sciences 10, no. 14 (2015): 5947-5953.

21. M. Kamal Narayanan, M. Karthik, K.Priya and R.M.Gomathi, Data Security Using Third Party Authority in Cloud Computing, International Conference on communication and Signal Processing (ICCSP), PP 1182-1185,Apr 2017

22. R. Abirami, S. Henitha, P. Saravanan, Fingertip Tracking Android Application for Hand Gesture Recognition, International Journal of Pharmacy and Technology, Vol. 8, No. 2, pp. 12028-12036, 2016 